\title{
First among equals: co-hyperintensionality for structured propositions
}

\author{
Bjørn Jespersen ${ }^{1,2}$ (D)
}

Received: 25 June 2020 / Accepted: 2 December 2020 / Published online: 26 December 2020

(c) The Author(s) 2020

\begin{abstract}
Theories of structured meanings are designed to generate fine-grained meanings, but they are also liable to overgenerate structures, thus drawing structural distinctions without a semantic difference. I recommend the proliferation of very fine-grained structures, so that we are able to draw any semantic distinctions we think we might need. But, in order to contain overgeneration, I argue we should insert some degree of individuation between logical equivalence and structural identity based on structural isomorphism. The idea amounts to forming an equivalence class of different structures according to one or more formal criteria and designating a privileged element as a representative of all the elements, i.e., a first among equals. The proposed method helps us to a cluster of notions of co-hyperintensionality. As a test case, I consider a recent objection levelled against the act theory of structured propositions. I also respond to an objection against my methodology.
\end{abstract}

Keywords Structured proposition · Co-hyperintensionality $\cdot$ Structural isomorphism $\cdot$ Equivalence class $\cdot$ Act theory

\section{Introduction}

Mary loves John. Mary is such that she loves John. Mary is one of those (maybe the only one) who love John. John is such that he is one of those (maybe the only one) that Mary loves. Mary and John, in that order, are such that the first one loves the second one.

Did we just say pretty much the same thing, though in different ways? Or does each of these sentences express something importantly different? My immediate

Bjørn Jespersen

b.t.f.jespersen@uu.nl; bjornjespersen@gmail.com

1 Department of Philosophy and Religious Studies, Utrecht University, Janskerkhof 13, 3512 BL Utrecht, The Netherlands

2 Department of Computer Science, VSB-Technical University of Ostrava, 17. listopadu 15, 70833 Ostrava, Czech Republic 
reason for asking this question is because a recent paper, Båve (2019), levels an objection against the act-theoretic conception of propositions, arguing that it fails to assign a unique meaning to "Mary loves John". I am convinced, however, that the objection can be rebutted. I am also convinced there is a general lesson in there for friends of structured propositions. This lesson bears on a methodological point that dispenses with a recurring problem for theories embracing structured propositions. The problem, in brief, is how to dial down the excessive fine-graining that afflicts structured propositions when allowed to generate distinctions that are not obviously, or obviously not, of semantic relevance. ${ }^{1}$ Here I will be making a case for the general form of the solution to the challenge posed in Båve (ibid.). The form of the solution carries over easily to theories of structured propositions other than just the various act-theoretic ones. To profit from the proposed solution, however, it is a requirement that the theory in question has already been developed formally, or else readily lends itself to being formally developed.

Let me be more specific. A common feature of theories of structured meanings is overgeneration of structures. Overgeneration is exactly the card Båve plays against the act-theoretic conception. This move makes sense for Båve to make, because he considers just two principles of individuation: (coarse-grained) logical equivalence and (very fine-grained) structural identity. However, he ought to have considered a third principle of individuation: (not overly fine-grained) structural isomorphism. ${ }^{2}$ The lesson for friends of all sorts of structured propositions is that there is a method to handle overgeneration. Here is how. First, allow an untrammelled proliferation of structured propositions in order to generate all the propositions you think you will ever need. Next, collect them into equivalence classes in accordance with one or more carefully selected criteria for membership. Finally, pick one member of each set as a representative of all of its fellow elements. This designated member is the structured proposition that is the meaning of the sentence which you originally set out to assign a meaning to.

The rest of the paper is organized as follows. Section 2 explains how the key notions are related. Section 3 presents Båve's objection. Section 4 dismantles this objection and sets out my positive contribution. Section 5 addresses an objection to my methodology.

\footnotetext{
1 The problem I am addressing in this paper is syntactic distinctions without a semantic difference. But a further issue is that a poorly-designed theory of hyperintensionality allowing too much fine-graining is at risk of engendering paradoxes. See Landini (2009), Cocchiarella (2007, 111ff). Also see Klement (2002, 112-13) on the violation of Cantor's theorem ('more sets than members') due to the Myhill-Russell paradox. Hyperintensional and set-theoretic paradoxes are topics I will not broach here, though, not least because it would involve us in a thorough discussion of logics/calculi that are either untyped or else developed within a simple type theory or a ramified type hierarchy.

2 Cf. Carnap's intensional isomorphism, Church's synonymous isomorphism, and the procedural isomorphism of Transparent Intensional Logic (see Duží 2019; Duží et al. 2010).
} 
The act-theoretic conception of propositions (or act theory, for short) holds that a proposition whose satisfaction conditions are truth-conditions is identical to a structured predicative act or event type. The atomic proposition that object $a$ has property $F$ is identical to the act type of predicating $F$ of $a$; the atomic proposition that objects $a, b$ are related by relation $R$ is the act type of predicating $R$ of $(a, b)$ or $\langle a$, $b\rangle$, depending on whether $R$ is symmetric; the molecular proposition Fa and Rab is the act type of predicating of the propositions $F a, R a b$ that they form a conjunctive proposition. $^{3}$

Båve claims to be able to generate a reductio of a fragment of act theory. He claims that any sentence containing a polyadic predicate such as 'loves' or 'is located between' induces structural ambiguity, which results in an unacceptable multiplication of propositions. I agree with Båve's observation about structural ambiguity, but I intend to show that the resulting sprawl of propositions need not be a problem for act theory or any other theory of structured propositions.

To begin with a monadic predicate, the sentences "Venus is a planet", "Venus is such that it is a planet", "Venus is something that is a planet" and "Venus is one of the planets" can be made, if one so desires, to come out synonymous, i.e., as notational variants of one another. The last three, to be sure, demand more logical processing or contain more logical steps than the first one. But a well-designed semantic theory of natural language should have the means to blot out such differences, in case it deems them insignificant. This is emphatically not to advocate that meanings should be individuated only up to necessary, or truth-conditional, equivalence; far from it. We definitely want to preserve various hyperintensional distinctions, e.g., in order to preserve various forms of topic and focus articulation so that we may keep track of what exactly is being asserted, or to specify the exact intellectual trajectory a particular agent follows when entertaining a particular attitude with a particular structure. For instance, it is arguably one thing to believe that Mary loves John and another thing to believe that Mary has the property of loving John. The latter attitude complement adds and highlights the notion of a property, and whereas the former can be construed as involving either a relation (loving being a relation extending from Mary to John) or a property (loving John), the latter rules out the relational reading. In fact, Båve's primary examples of structural ambiguity are examples of hyperintensional distinctions we do want to preserve. But too much fine-graining is too much of a good thing. So, what we are basically looking for is one or more reasonable and operative criteria of co-hyperintensionality, such that

\footnotetext{
3 Vide Hanks (2015), Soames (2019). A referee wondered whether the act-theoretic account of conjunctive propositions requires several layers of predicative acts. I myself find the act-theoretic conception of molecular propositions rather problematic, and my (2021) explains why even conjunctive propositions are tricky. Still, the act theorist is not simply nesting assertoric predications within assertoric predications. The official explanation, at least, according to Hanks is that target-shifted predication, $\vdash_{\uparrow}$, serves to mention the act type $F a$ and the act type $R a b$ without engaging in two assertoric acts, and CONJ is the act type that conjoins those two atomic act types into the molecular act type of asserting both of them in a particular order. The act-theoretic formula looks like this: $\boldsymbol{F}_{\uparrow}\langle(\boldsymbol{H}\langle\mathbf{a}, \mathbf{F}\rangle, \mathcal{H}\langle\langle\mathbf{a}, \mathbf{b}\rangle, \mathbf{R}\rangle), \mathbf{C O N J}\rangle$.
} 
any two co-hyperintensional structured propositions become members of an equivalence class sporting a privileged member. ${ }^{4}$

Bear in mind that hyperintensionality was originally negatively defined: any individuation that defies the identification of logical equivalents is hyperintensional. This leaves open the question what is the upper bound to hyperintensional individuation, which translates into the quest for criteria of co-hyperintensionality. Only when we have availed ourselves of a criterion of co-hyperintensionality can we develop a notion of validity for hyperintensional contexts. For then we are not only blocking (invalidating) undesired arguments, but also enabling (validating) inferences we do want to go through. At such a point we will have arrived at a hyperintensional logic. ${ }^{5}$ When hyperintensions are structured entities, the task becomes how to formulate a principle of structural isomorphism that defines co-hyperintensionality for structurally diverse, but otherwise relevantly identical, structures. My diagnosis is that Båve's attempted reductio is predicated on ignoring structural isomorphism and focusing exclusively on structural diversity.

\section{3}

I describe here how Båve puts his attempted reductio together. Asymmetric polyadic predicates are delicate, because the order of the relata matters, so I will stick to such examples. First, Båve (ibid., 183) claims that

"Mary loves John"

can be analysed in three different ways:

(ii) as the result of saturating " $x$ loves John" with 'Mary'

(iii) as the result of saturating "Mary loves $y$ " with 'John'

(iv) as the result of saturating " $x$ loves $y$ " with 'Mary' and 'John' in the first and second argument place, respectively.

\footnotetext{
${ }^{4}$ For recent surveys of strategies for obtaining fine-graining and various degrees of granularity, see Faroldi (2017), Sedlár (2019), Giordani and Malinowski (2021, 1-11). See Ayhan (2020, esp. §2.2.) on forming equivalence classes (under various conversion rules from the $\lambda$-calculus), in this case of derivations, so as to define identity criteria for logical proofs.

${ }^{5}$ Various theories embracing hyperintensionally individuated entities are keen to include a separate level of intensionally individuated entities, where co-intensionality is tantamount to logical equivalence. Examples would be Bealer's type 1 and type 2 PRP's, i.e., propositions, relations, and properties (1982), Cocchiarella's two levels (2007), and the constructional versus intensional level in Transparent Intensional Logic (Duží et al. 2010). All three positions reserve, roughly speaking, the hyperintensional level for attitudes and assertions and the intensional level for modalities and empirical states-of-affairs (though some modalities, most notably counterpossibles, are definitely hyperintensional). These two levels are logically connected. The approach characteristic of such two-tiered theories is that the hyperintensional level is not deductively closed, whereas the truth-conditional/intensional level is: here are located the deductive consequences of, e.g., believed and asserted hyperpropositions.
} 
Each of these three ways of saturating the argument slots corresponds to an alternative analysis of "Mary loves John". 6 Next, he introduces the family of functions $f_{n}$ where $f_{n}$ takes so-called semantic correlates of terms and expressions to structured propositions and where $n$ indicates the arity of the predicate involved. Banve later replaces $f_{n}$ by Zalta's plug, which he deploys as a device to model the act type of substituting the semantic correlate of a name for the value of a variable. For instance, $f_{1}$ takes the semantic correlates $[F(x)]$ and $[a]$ of the monadic predicate " $\xi$ is an $F$ ' and the name ' $a$ ' to the semantic correlate $[F(a)]$ of the sentence " $a$ is an $F$ '. Correspondingly, $f_{2}$ takes the semantic correlates of a dyadic predicate and two names to a proposition composed of them (see ibid., 187).

Finally, Båve attempts to set act theory up for a reductio. This is the key passage:

[...] a sentence ' $\mathrm{Fa}$ ' must be taken to express a [structured] proposition composed of the semantic correlates of ' $F(\xi)$ ' and ' $a$ ', that is, $[F(\xi)]$ and $[a] .[\mathrm{F}(a)]$ is thus the value of some function $f$ from $[F(\xi)]$ and $[a]$; that is, $[F(a)]=f([F(\xi)],[a])$. But for a sentence like 'Mary loves John', there are two choices for the respective ' $[F(\xi)]$ ' and ' $a$ ' [i.e. ' $[R(\xi, \zeta)]$ ', ' $a$ ', ' $b$ '. BJ.]. Thus, its semantic correlate, [Mary loves John], must be identical to both $f$ ([Mary loves $\xi]$, [John]) and $f([\xi$ loves John], [Mary]). And this is just what it means for a proposition to have alternative analyses. (Ibid., 186.)

This may be a bit hard to parse, so I will explain what is going on. Båve's point is that $f$ takes two different arguments, namely ([Mary loves $\xi]$, [John]) and

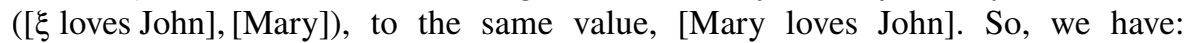
$[$ Mary loves John $]=f([$ Mary loves $\xi],[$ John $])=f([\xi$ loves John $]$, [Mary $])$.

The problem, as Båve sees it, is that act theory creates room for two equally good contenders to being the proper way of saturating slots such that a slot-free (i.e., saturated) formula (i.e., a sentence) is generated. ${ }^{7}$ The sentence "Mary loves John" expresses as its meaning a structured proposition composed of the respective semantic correlates of ' $[$ Love $(\xi, \zeta)]$ ','Mary','John'. But, Båve wonders, is that proposition $f([$ Mary loves $\xi]$, [John] $)$ or is it $f([\xi$ loves John], [Mary])? Which of these two structures is the structure of [Mary loves John]? This question supposedly places act theory before a dilemma that is entirely of act theory's own making. The intended reductio is that the act theorist has no non-arbitrary way of selecting one structure as the structure of the act type that is the meaning of "Mary loves John". Absent such a selection procedure, there is an uncalled-for structural ambiguity, hence an uncalledfor proliferation of structures, i.e., act types, i.e., propositions.

To get a handle on how exactly Båve's intended reductio goes, I suggest turning to the lingua franca of natural-language semantics, namely the $\lambda$-calculus, for a

\footnotetext{
${ }^{6}$ Båve ought to have phrased his point in terms of alternative decompositions rather than analyses. As Levine (2002) stresses, any analysis is a unique analysis, with only simple constituents left, whereas no one decomposition is required to terminate in rock-bottom atoms. Båve's point can be made more forcefully in case there is no analysis/decomposition with a unique feature (such as identifying all the simple constituents) to have recourse to.

7 The passage above leaves out (iv), but the availability of just two different structures already suffices to make his point.
} 
more neutral symbolism that is well-understood. ${ }^{8}$ In this calculus, [Mary loves John] emerges as the contractum of multiple equivalent reduxes. The contractum receives this form:

(i*) $L m j$

The three 'alternative analyses' Båve mentioned at the outset match the following $\lambda$-abstracts:

(ii*) $(\lambda x(L x j)) m$

(iii*) $\quad(\lambda y(L m y)) j$

$\left(\mathrm{iv}^{*}\right) \quad(\lambda x y(\mathrm{~L} x y))\langle m, j\rangle$

In fact, why stop right here? For now, we are considering only proliferation and not also restriction. Thus, we are free to generate formulas/structures such as these ${ }^{9}$ :

$\left(\mathrm{v}^{*}\right) \quad(\lambda x(\lambda y(L x y)) j) m$

(vi*) $\quad(\lambda y(\lambda x(L x y)) m) j$

Or, if the logical ideography in question allows $\lambda$-binding predicate variables and allows predicates to occur in subject position ("Being wise is wonderful"), we can carry the proliferation even further by generating a formula/structure like this one:

(vii*) $(\lambda x y((\lambda r(r x y)) L))\langle m, j\rangle$

The formula expresses that $L$ is one of the (binary and asymmetric) relations between individuals $x, y$ such that $L$ applies to $m, j$; or, equivalently, that $L$ is a (binary and asymmetric) relation between individuals such that it relates $x, y$ and $m$, $j$ are such that they are values of $x, y$ whereby $L$ relates them. From here, we could carry on generating abstracts by iterating $\lambda$-abstraction at our pleasure. Only doing so would be philosophically pointless because these abstracts have no natural natural-language counterparts (we would most likely just have strings of increasingly contorted 'such that' constructions). The reduxes above, however, do have such counterparts as they highlight different topics and foci. ${ }^{10}$ For instance, while ( $\left.i^{*}\right)$ is neutral or plain, (ii*) makes $m$ the topic, (iii*) makes $j$ the topic, (iv*) makes the pair $\langle m, j\rangle$ the topic, and (vii*) makes $L$ the topic.

Before moving on, I want to say a bit more about the shift from Frege's to Church's logic and ideography as encapsulated in the $\lambda$-calculus. Notice that Båve's argument based on Frege-style complex predicates, with saturation of slots, is

\footnotetext{
${ }^{8}$ Hanks's act-theoretic notation for "Mary loves John" would be (cf. Hanks $\left.(2015,85)\right)$ : $-\langle\langle$ Mary, John $\rangle$, LOVES $\rangle$. However, the syntax is not accompanied by a logic, so we would not know how to draw inferences by means of it.

9 I am indebted to a referee for suggesting $\left(\mathrm{v}^{*}\right),\left(\mathrm{vi}^{*}\right)$.

10 Båve claims, "A fortiori,"Mary loves John" does not seem to "say" different things depending on the "parsing"." (Ibid., 195) But it very much does. The truth-conditions are the same, for sure, provided we rule out truth-value gaps, but the seven 'parsings' $d o$ 'say' different things, namely in virtue of topic/ focus articulation.
} 
nothing new. (What is new is the application specifically to act theory.) For instance, Tichý (1986, 519-20) asks which function Frege has '-' denote in '9-2': (i) the function of subtracting the second argument from the first, $\xi-\zeta$, or (ii) the function of subtracting the first argument from the second, $\zeta-\xi$. This is important, (i) and (ii) being distinct functions (whether unsaturated Fregean ones or modern-day mappings). If the question is left unanswered, the result is that '-' comes out two-way ambiguous. In fact, we can even generate three-way ambiguity with ' $2-2$ ': $\xi-\zeta$, $\zeta-\xi, \xi-\xi$. This ties in with a passage in Klement $(2002,104-05)$ :

The possibility of 'alternative analyses' or subject/predicate renderings of the same Gedanke [...] is a consequence of functional comprehension. Frege, too, is committed to functions existing for each of Church's lambda abstracts. For Church's ' $(\lambda x x>7)$ ', Frege has simply ' $\xi>7$ ', and for Church's ' $(\lambda x 9>x)$ ', Frege has ' $9>\zeta$ '. The difference, however, is that Frege's notation clearly shows the unsaturatedness of these functions, whereas Church's does not [nor did Church intend his ideography to do so, of course. BJ]. The notation " $(\lambda x x>7) 9$ " would seem wholly unnatural to Frege; it would be akin to writing " $(\xi>7) 9$ ", which would only obscure the fact that the Sinn of ' 9 ' is thought to complete the incomplete Sinn of ' $\xi>7$ ' to form a whole, and, in so doing, it eliminates the unsaturatedness marked by ' $\xi$ '. The resulting Gedanke is better expressed simply as " $9>7$ ". Note that the same whole is formed when the incomplete Sinn of ' $9>\zeta$ ' is completed by the Sinn of ' 7 '.

Klement (ibid.) goes on to argue that

Frege would transcribe both Church's " $(\lambda x x>7) 9$ " and his " $(\lambda x 9>x) 7$ " simply as " $9>7$ ". This too leads us to the conclusion that Frege would see no difference in the Sinne of lambda converts, and thus that we ought to favor Alternative (1) over Alternative (0) as the more Fregean alternative [...].

Again, this is so because Frege, on Klement's reading, would have had little time for $\lambda$-abstracts in the first place. ${ }^{11}$ Note, however, that the (hypothesized) aversion against $\lambda$-abstracts rests on the theory-internal reason that Frege wants his ideography to signal the unsaturatedness of his functions. If we are agnostic about unsaturatedness then we are free to translate a predicate like ' $\xi>7$ ' into ' $\lambda x(x>7)$ '. We are also free not to agree with Frege's (hypothesized) misgivings about $\lambda$-abstracts. Thus, though Båve frames the problem of alternative analyses (or decompositions) explicitly in terms of saturation, his point about structural ambiguity can be made equally well in terms of variables and functional application/abstraction.

\footnotetext{
11 For a comparison of Church's three alternative interpretations of synonymous isomorphism - (A0), based on $\alpha$-conversion together with meaning postulates for semantically simple terms; (A1), based on $\beta$-conversion; and (A2), based on logical equivalence - see Klement (ibid., 101-11) and Anderson (2001), which also considers an intermediate alternative. $\alpha$-conversion is just uniform renaming of $\lambda$-bound variables; e.g., $(\lambda x(L m x)) j={ }_{\alpha}(\lambda y(L m y)) j . \alpha$-conversion is often a prelude to $\beta$-conversion in order not to get entangled in collision of variables, and the result of correct conversion is known as $\alpha \beta$-identity.
} 
Båve argues at length that the alternative analyses he puts forward are distinct act types, and I agree. Accordingly, we have, for instance, $f_{2}$ ([Mary loves $\left.\xi\right],[$ John] $) \neq_{h} f_{2}\left(\left[\xi\right.\right.$ loves John], [Mary]), where ' $={ }_{h}$ ' expresses the hyperintensional identity (as opposed to truth-conditional equivalence) relation between structures. The differently structured propositions $f_{2}$ ([Mary loves $\left.\xi\right]$, [John]) and $f_{2}$ ([ $\xi$ loves John], [Mary]) are co-intensional (because they converge in the same truth-condition) at those two arguments, despite the respective arguments being distinct. The same function takes two distinct arguments to the same value, because such is the effect of these two instances of saturation (or substitution) as per $f_{2}$. When Båve speaks of identity in the quote above, he obviously intends coarser-grained (viz., truth-conditional) identity, as in: $f_{2}$ ([Mary loves $\left.\xi\right]$, [John] $)={ }_{e q} f_{2}([\xi$ loves John], [Mary]). Otherwise he would be simultaneously arguing for alternative (i.e., multiple) analyses and identity (i.e., singularity) between them, which would defy his reductio.

I think we can now appreciate the point Båve aims to make. When he claims there is identity between the alternative analyses and ( $\left.{ }^{*}\right)$, Båve's point is that the contractum can be equivalently and equally well generated, by way of $\beta$-conversion, from any of these reduxes and that the act theorist is at a loss as to which formula (hence which corresponding semantic correlate, i.e., structured proposition) to select as the meaning of "Mary loves John". Obviously, no semantic theory wants to assign more than one structurally determinate proposition to a lexically and structurally unambiguous sentence as its meaning (or semantic correlate). If this were the end of it, Båve's attempted reductio would be successful.

\section{4}

But it is not, provided the act theorist is prepared to adopt my proposal. The awkward question is supposed to be this: which of (i*) through (vii*) is the meaning of "Mary loves John"? Or as Båve dramatizes the problem:

But which of the [six] propositions expressed by ["Mary loves John"] do we believe when, as we would naïvely put it, we "believe that [Mary loves John]"? [All six of them?] Could one believe one but not the [rest]? (Ibid., 195. I stuck with the original rather than Båve's additional example.)

My answer is: pick the one whose logical form matches the syntactic form of the sentence the closest. If the analysandum is "Mary loves John" then you pick (i*) Lmj. If the analysandum is along the lines of "Mary is such that she loves John", "Mary is someone who loves John" or "Mary is among those who love John" then you pick (ii*) $(\lambda x(L x j)) m$, i.e. $f_{2}([\xi$ loves John], [Mary $])$.

What the act theorist should not do is join their detractor in treating the various analyses as being on an equal footing. The right thing to say is that "Mary loves John" means Lmj, and that it is a further question which other structures Lmj is structurally isomorphic to. That is, the meaning of "Mary loves John" is nowhere 
close to being structurally ambiguous. (ii*) through (vii*) are the respective meanings of six different sentences, which are mutually truth-conditionally equivalent, but not synonymous, because ( $\left.\mathrm{ii}^{*}\right)$ through (vii*) are not hyperintensionally identical. Or to answer Båve's question head-on: no, we do not believe all six of them at the same time, for the doxastic relation combines a believer and one proposition. Of course, if belief is coarse-grained and, thus, individuated only up to truth-conditional equivalence then any of $\left(i^{*}\right)$ through (vii*) can serve equally well as complement. But if, on the other hand, belief is very fine-grained and individuated by dint of structural identity ( $c f$. inscriptionalism or sententialism), then only (i*) will do. What we believe, ex hypothesī, is that Mary loves John, pure and simple, without any part being highlighted.

If, as I recommend, the act theorist embraces Båve's ambiguity, the two remaining tasks to be completed are (a) defining an equivalence class of act-theoretic propositions, and $(b)$ designating one member as a primus inter pares to serve as the single meaning of a sentence and the complement of a hyperpropositional attitude.

We begin with (a), which is to some degree anticipated in Båve (ibid., 199). He rejects identifying propositions with equivalence classes of act types as a viable 'retreat position' for act theory. On this proposal, the various alternative analyses (decompositions) of [Mary loves John] are collected into a set, and [Mary loves John] is then identified with this set. Båve has two objections to this, both of which are legitimate: "this [retreat position] does not take propositions to be (syntactically) structured", and it fails to "[distinguish] logically equivalent propositions without thereby multiplying propositions wherever there are alternative analyses." The remedy he suggests is to make the criterion of equivalence so austere that only structurally identical propositions qualify as equivalent. But, as he in effect says, we then lose many equivalences we would want to maintain.

What stymies this retreat position is that [Mary loves John] is identified with a set of (however loosely or austerely) equivalent propositions instead of being a privileged member of a set of equivalent propositions. If we go with the latter approach, we select a criterion of co-hyperintensionality that blots out structural differences between pairs of co-hyperintensional propositions when those are theoretically irrelevant. We trim the fat, as it were. If we stick to (ii*), (iii*) for a toy example, they form the equivalence class $\{(\lambda x(L x j)) m,(\lambda y(L m y)) j\}$ under $\beta$-conversion, assuming, strictly for the sake of exposition, that this conversion rule constitutes the criterion of structural isomorphism for propositions. Such an equivalence class is generated by applying a conversion rule (or a cluster of conversion rules) to the structure underlying an input sentence such as "Mary loves John". The members are structurally isomorphic in the sense that they convert to one another under the chosen conversion rule (or cluster thereof). The different $\lambda$-formulas that represent the structured propositions which are members of the equivalence class come with different syntactic structures, but the conversion rule(s) generating the class dictate(s) that these syntactic differences are 
semantically insignificant. ${ }^{12}$ Co-hyperintensionality is now predicated on structurally distinct hyperintensions being structurally isomorphic. Thus, co-hyperintensionality remains fine-grained, as it should, but gets a little coarser, as it also should. Structural isomorphism is the position tucked in between structural identity and logical equivalence that Båve failed to consider. The (right) answer to Båve's question above as to what we believe when we believe that Mary loves John now becomes instead: $\left(i^{*}\right)$ or any other proposition structurally isomorphic to it; for any pair of such propositions is freely substitutable within this hyperintensional context.

Since I am making a methodological rather than substantial point in this paper, my only concern is to settle for some particular rule (or set of rules) of conversion in order to arrive at an equivalence class, and not to push for this or that particular rule (or set of rules). However, we still need to pause to ponder the special role $\beta$-conversion plays in Båve's argument against act theory. This is $\beta$-conversion:

$$
\left(\lambda x_{1}, \ldots, x_{n} A\left(x_{1}, \ldots, x_{n}\right) y_{1}, \ldots, y_{n}\right) \approx_{\beta} A\left(y_{1}, \ldots, y_{n}\right)
$$

where each $y_{i}$ is free for each $x_{i}$ in $A$. Remember that (ii*) through (vii*) $\beta$-reduce to $\left(i^{*}\right)$. So in case $\beta$-conversion is deemed too coarse as a reasonable criterion of co-hyperintensionality then Båve's identification - i.e., $[$ Mary loves John $]=f([$ Mary loves $\xi],[$ John $])=f([\xi$ loves John $],[$ Mary $]) \quad-\quad$ cannot be generated and so Båve can no longer place act theory before the dilemma of having to select one of $f$ ([Mary loves $\xi]$, [John]), $f([\xi$ loves John], [Mary]) at the expense of the other. Apart from wanting to evade the dilemma, there is independent reason, anyway, for banning $\beta$-conversion as a suitable criterion of co-hyperintensionality for structured propositions. Already the three structures of $L m j,(\lambda x(L x j))$ $m$, and $(\lambda y(L m y)) j$ are arguably so distinct that it seems an open-and-shut question which one must be the structure of the meaning of "Mary loves John", and that is $L m j$, as I have been arguing. $L m j$ has a logical connection with $(\lambda x(L x j)) m$ and $(\lambda y$ $(L m y)) j$, for sure, but neither of them enters the picture as a candidate structure for the meaning of "Mary loves John". ${ }^{13}$ Pointing out as much could, narrowly speaking, be the right rejoinder to Båve's charge of ambiguity. However, this sort of reply would miss the more profound issue implicit in Båve's challenge. And that is how

\footnotetext{
12 See Duží (2019), Jespersen (2015), Salmon (2010) for a discussion of whether to include $\beta$-conversion (and if so, which particular variant, i.e., by-name or by-value) or other conversion rules of the $\lambda$-calculus to get the calibration of co-hyperintensionality right for this or that particular sort of context.

13 Barendregt $(1991,20)$ notes an asymmetry in $\beta$-reduction that is relevant to my line of reasoning: $(\lambda x$ $\left.\left(x^{2}+1\right)\right) 3=10$ can be understood as 10 being the result of computing $\left(\lambda x\left(x^{2}+1\right)\right) 3$, but not vice versa. Likewise, I would say, $(\lambda x(L x j)) m=L m j-$ or $(\lambda y(L m y)) j=L m j$ - can be understood as $L m j$ being the result of computing $(\lambda x(L x j)) m-$ or $(\lambda y(L m y)) j-$ though not vice versa. But note the following. If one considers an equivalence class under $\beta$-conversion then the relation between any two $\beta$-converts in this class can be described as one of material equivalence. The reason is that the function (provided we remain within a calculus of total functions) yielding the various $\beta$-converts is one and the same. Yet at the (hyperintensional) level of computation, $\beta$-conversion is asymmetric, as Barendregt emphasizes. Once a series of computations has terminated in the normal form - here, $L m j$ or 10 - then there is no trace of how this form was obtained. For instance, in Duží and Jespersen (2013) we revisit the old verbellipsis cases such as "John loves his wife, and so does Peter" and show that the application of two different properties - loving one's wife versus loving John's wife - to John followed by $\beta$-reduction-by-name reduces the first conjunct to "John loves John's wife" in both instances, leaving it indeterminate which of
} 
to allow propositional structures to stray from the grammatical structure of a given sample sentence, i.e., to allow proliferation of propositional structures, without generating structural ambiguity. Enter structural isomorphism.

However, invoking structural isomorphism requires taking a closer look at complex predicates. ${ }^{14}$ The particular structure of a proposition will be in part a function of the property or relation being predicated. Therefore, this question arises: must any two co-intensional predicates that exhibit different syntactic structures map onto two structurally distinct (hyper-) properties (ignoring relations for now), which in turn yield the same evaluation function? For instance, should is someone who is someone who is such that they love John' denote a property different from what 'loves John' denotes? The worry lurking in the background is that we do not want to be saddled with a bloated ontology populated by (hyper-) properties some of whose differences in structure are without semantic significance. Any such ontological excess among the semantic values of predicates will devolve onto structured propositions once such predicates are used to predicate properties of individuals. One could rightly object that a property whose logical structure matches perfectly (as far as $\lambda$-abstracts will allow) the syntactic structure of is someone who is someone who is such that they love John' has no business being in the structured proposition that Mary loves John. ${ }^{15}$ But, for instance, in a theory such as Transparent Intensional Logic the syntactic differences between two predicates do map onto two different objective logical structures; these in turn yield mappings each of which has a task to fulfill in determining the satisfaction conditions denoted by the predicates in question. (Other theories may opt for syntactic complexes combined with metaphysical simples, as in Bealer.) Still, there is a way to temper this proliferation of objective structures. Those two syntactically distinct predicates will co-denote the same property (i.e., mapping of empirical indices onto sets of individuals), while each also expressing a different conceptualization of it. That is, we are looking at two differently structured itineraries (i.e., hyperintensions) travelling toward the same satisfaction condition (i.e., intension). Those two predicates will come out co-intensional without being co-hyperintensional. Consequently, being someone who is someone such that they love John and loving John will be one and the same property. But this property cannot be a constituent of a structured proposition, according to, e.g., Transparent Intensional Logic; a particular conceptualization, with a particular logical structure, of it can. This particular structure will affect the overall structure of the proposition, and we already know what the method is for obtaining the desired degree of propositional granularity.

\footnotetext{
Footnote 13 (continued)

the two properties is to be picked up by 'so does' in the second conjunct. On one reading, both John and Peter are exemplary husbands. On the other reading, there is trouble on the horizon.

14 I am indebted to a referee for pressing me on this point.

15 Pursuing this topic more rigorously requires taking a stand on the semantic import of $\eta$-conversion: $\lambda x(f x) \approx_{\eta} f$, provided $x$ is not free in $f$. But I will leave it at that for now. See, however, Ayhan (2020, fn. 6) for discussion and references
} 
We move on to $(b)$. Which of the three members of $\{L m j,(\lambda x(L x j)) m,(\lambda y(L m y)) j\}$ should be privileged as a first among equals? A natural guideline, which I already applied above, is that we want the best approximation we can get to a match between sentential and propositional structure, because structure serves to specify the sequence in which we process meaning. For instance, the syntactically unadorned "Mary loves John" enjoins us to simply arrange Mary and John in the loving relation, with Mary in subject and John in object position, as the loving relation extends from Mary to John. And that is all the sentence does, so our pick is Lmj. Topic/focus articulation will systematically affect the selection of the privileged member, but the unadorned "Mary loves John" (unaccompanied by pitch emphasis when spoken) is merely concerned with getting the truth-condition right and so is characterized by being insensitive to topic/focus articulation.

My pick of Lmj runs counter to Båve's reason for claiming (ibid., 183) that "Mary loves John" is analyzable into multiple act types (or multiple structures, more generally). Of course, "Mary loves John" is so analyzable, but that is irrelevant. In my view, the structures $(\lambda x(L x j)) m$ and $(\lambda y(L m y)) j$ are the respective meanings of other sentences along the lines of "Mary is someone who loves John" and "John is someone whom Mary loves". In fact, the differences between these three structures are hard to miss. "Mary loves John" expresses the application of the binary relation of loving to the ordered pair 〈Mary, John〉. "Mary is someone who loves John" expresses the application of the property of loving John to Mary. "John is someone whom Mary loves" expresses the application of the different property of being loved by Mary to John. To be sure, a formal semanticist who has convinced themselves that $\beta$-conversion defines structural isomorphism is going to claim that these three structures are co-hyperintensional. And in this case we are facing one meaning rather than three. But I have been trying to argue why $\beta$-conversion is too coarse for the purposes of structural isomorphism.

\section{5}

The above policy of proliferation of structured propositions appears vulnerable to the objection that it trivializes the task of assigning meanings to sentences. The policy predicts a more-or-less bijective relationship between sentences and propositions, whereas (so the objection goes) the mapping ought to be surjective, taking multiple sentences to the same proposition. Some differences in sentential structure should not go over into differences in propositional structure. If, on the other hand, propositional structure tracks, by and large, sentential structure, then the semanticist barely gets to make fallible claims about sentence/meaning pairs, because any two distinct sentential structures will have already been mapped into two different propositional structures. Barely any pairs of sentences will, therefore, occur in the synonymy relation. An objection along these lines is put forward in Pickel (2018), which targets not only Jeffrey King's theory, which has proliferation of propositional structures as an accidental by-product, but also a theory such as mine, which actively encourages proliferation. What does commend a pro-proliferation approach, according to Pickel, is this: 
[It offers] a maximally flexible framework [...] For instance, [when sentences $S, S^{*}$ have already been deemed to express different propositions $\llbracket S \rrbracket$, $\left[S^{*} \rrbracket\right]$ it might be found that there is a construction $\sum$ (.) such that the observational data support $\left[\left[\sum(S)\right]\right] \neq\left[\left[\sum\left(S^{*}\right)\right]\right]$. This data will be easier to accommodate if we already have that $\llbracket S \rrbracket \neq \llbracket S^{*} \rrbracket$. (Ibid., Sect. 2, fn. 12.)

If instead the (less-profligate) semanticist had ventured forth with the claim that $\llbracket S \rrbracket=\llbracket S^{*} \rrbracket$ then the truth of $\left.\left[\left[\sum(S)\right]\right] \neq \llbracket\left[\sum\left(S^{*}\right)\right]\right]$ would constitute a counterexample. One would learn from this the non-trivial truth that $\llbracket S \rrbracket \neq \llbracket S^{*} \rrbracket$, which would emphatically not be an artefact of one's semantics. ${ }^{16}$

What to make of Pickel's objection? I think it is spot on. The concept of synonymy is central to any formal semantics worth its name, because it regulates such things as equality of expressive power and the substitutability of sentences in hyperpropositional attitude reports. Any logically and philosophically interesting mapping from a domain of sentences onto a range of propositions must obviously be a surjective one. But it is exactly for this sort of reason that proliferation is only the first half of my recommended approach. The second half consists namely in tightening the noose by means of equivalence classes and the appointment of representative members. ${ }^{17}$

This reply to the objection is incomplete, however, without a satisfactory answer to this question: which formal criterion should we pick? My answer is this. The choice of criterion calls for philosophical discretion. Different sorts of hyperintensional contexts are likely to be different not least by calling for different degrees of fine-graining. ${ }^{18}$ Imagine we are confronted with a context which our philosophical considerations have convinced us requires a certain granularity to validate certain inferences and block certain other inferences (often dressed up as puzzles by which to measure the mettle of rivalling theories). Then we consult our abundant supply of structured meanings and superimpose a formal criterion (e.g., again strictly for the purpose of exposition, $\alpha$-conversion paired with $\eta$-conversion), which yields a class

\footnotetext{
16 Pickel's appeal to counterexamples seems to presuppose that the semanticist cannot have recourse to some form of Frege-Church-Montague-style contextualism, which would accommodate $\llbracket S \rrbracket=\llbracket S^{*} \rrbracket$ and $\left[\left[\sum(S)\right] \neq \llbracket\left[\sum\left(S^{*}\right)\right]\right]$ within the same semantic theory. On the topic of counterexamples, I readily grant that the methodology I am recommending lends itself to the sort of self-immunization Popper famously warned against. A semanticist facing a looming falsification might, for instance, be tempted to deny that the problematic context is actually of the sort governed by the chosen criterion of granularity. But we also know why the temptation should not be yielded to, because the argument from trivialization will eventually kick in.

17 This second half appears still to be absent from (or, at the very least, underdeveloped in) King's theory, which is marred by excessive fine-graining, as Pickel correctly notes in (ibid., fn. 11).

18 See the catalogue of degrees of granularity in Duží (2019). Likewise, Miller (2017) operates with two tiers of hyperintensional individuation in order to enable bearing different propositional attitudes ('weak hyperintensional distinction') to metaphysically equivalent theories ('strong hyperintensional equivalence'). An alternative path to weak hyperintensional distinction Miller brings up turns on lexical differences between, e.g., constants (so that Lois can believe that Superman is amazing without believing that Clark Kent is amazing). However, the relevance to structurally diverse propositions is only indirect, and my proposal above does not tamper with the constants and predicates involved, but basically makes do with increasing or decreasing the levels of logical processing.
} 
with a privileged element. Our choice of criterion leads us to make fallible predictions, as decreed by Pickel, and after canvassing a series of test cases we may well want to reconsider our initial choice.

There is no one sacrosanct, or absolute, measure of co-hyperintensionality. Structural isomorphism is not one particular measure of granularity, but rather a cluster of such measures. Structural isomorphism is a form whose content is this or that specific criterion given by one or more conversion rules.

\section{Conclusion}

I have extracted a general lesson from my solution to a specific objection. The objection was that a certain theory of structured propositions is stuck with structurally ambiguous sentences where no such ambiguity is called for. The first step toward a solution was to point out that there is a position tucked in between structural identity and logical equivalence, namely structural isomorphism. The next step was to argue that all theories of structured propositions (or structured meanings, in general) should go for maximal multiplication of their respective structures, but then do the following: $(i)$ provide philosophical motivation for the choice of a specific formal criterion of individuation for a particular kind of context, (ii) apply this criterion to one's rich supply of structures to extract an equivalence class of structurally isomorphic structures, (iii) designate a representative element as a first among equals, which is then the meaning of the sentence (or piece of language, in general) that one set out to assign a meaning to. This general approach to how a theory of structured meanings can profit from proliferation of structures does presuppose that the theory in question is susceptible to formal treatment, which includes one or more formally precise criteria of individuation of structures. A theory of structured meanings that is not yet sufficiently developed so as to rigorously individuate and identify its structures is liable to be embarrassed by the objection from overgeneration. ${ }^{19}$

Funding Grant no. GA18-23891-S of the Grant Agency of the Czech Republic.

\section{Complaince with ethical standards}

Conflict of interest The authors declare that they have no conflict of interest.

Open Access This article is licensed under a Creative Commons Attribution 4.0 International License, which permits use, sharing, adaptation, distribution and reproduction in any medium or format, as long as you give appropriate credit to the original author(s) and the source, provide a link to the Creative Commons licence, and indicate if changes were made. The images or other third party material in this article are included in the article's Creative Commons licence, unless indicated otherwise in a credit line to the

\footnotetext{
${ }_{19}$ I am much obliged to Marie Duží, Rachel Boddy and two anonymous referees for Synthese for very helpful comments on previous versions. This research was funded by Grant Agency of the Czech Republic project No. GA18-23891S, Hyperintensional Reasoning over Natural Language Texts, and Internal Grant Agency of VSB-Technical University of Ostrava project No. SGS No. SP2020/62, Application of Formal Methods in Knowledge Modelling and Software Engineering III.
} 
material. If material is not included in the article's Creative Commons licence and your intended use is not permitted by statutory regulation or exceeds the permitted use, you will need to obtain permission directly from the copyright holder. To view a copy of this licence, visit http://creativecommons.org/licen ses/by/4.0/.

\section{References}

Anderson, C. A. (2001). Alternative (1*): A criterion of identity for intensional entities. In C. A. Anderson \& M. Zeleny (Eds.), Logic, Meaning and Computation (pp. 393-427). Dordrecht: Kluwer Academic Publishers.

Ayhan, S. (2020). What is the meaning of proofs? Journal of Philosophical Logic. https://doi. org/10.1007/s10992-020-09577-2.

Barendregt, H. (1991). Lambda Calculi With Types. Retrieved from https://ttic.uchicago.edu/ dreyer/ course/papers/barendregt.pdf.

Bealer, G. (1982). Quality and Concept. Oxford: Clarendon Press.

Båve, A. (2019). Acts and alternative analyses. Journal of Philosophy, 116, 181-205.

Cocchiarella, N. (2007). Formal Ontology and Conceptual Realism, Synthese Library 339. Dordrecht: Springer-Verlag.

Duží, M. (2019). If structured propositions are logical procedures then how are procedures individuated? Synthese, 196, 1249-1283.

Duží, M., \& Jespersen, B. (2013). Procedural isomorphism, analytic information, and $\beta$-conversion by value. Logic Journal of the IGPL, 21, 291-308.

Duží, M., Jespersen, B., Materna, P. (2010). Procedural Semantics for Hyperintensional Logic, Heidelberg et al:: Springer-Verlag.

Faroldi, F. L. G. (2017). Co-hyperintensionality. Ratio, 30, 270-287.

Giordani, A., \& Malinowski, J. (2021). Logic in high definition - trends in logical semantics. In A. Giordani \& J. Malinowski (Eds.), Logic in High Definition, Trends in Logic (Studia Logica Library) 56 (pp. 1-11). Dordrecht: Springer-Verlag.

Hanks, P. (2015). Propositional Content. Oxford: Oxford University Press.

Jespersen, B. (2021). 'Two tales of the turnstile', IfCoLog Journal of Logics and their Applications, forthcoming.

Jespersen, B. (2015). Should propositions proliferate? Thought, 4, 243-251.

Klement, K. C. (2002). Frege and the Logic of Sense and Reference. New York, London: Routledge.

Landini, G. (2009). Cocchiarella's formal ontology and the paradoxes of hyperintensionality. Axiomathes, $119,115-142$.

Levine, J. (2002). Analysis and decomposition in Frege and Russell. Philosophical Quarterly, 52, $195-216$.

Miller, K. (2017). A hyperintensional account of metaphysical equivalence. Philosophical Quarterly, 67, $772-793$.

Pickel, B. (2018). Structured propositions and trivial composition. Synthese. https://doi.org/10.1007/ s11229-018-1853-1.

Salmon, N. (2010). Lambda in sentences with designators: an ode to complex predication. Journal of Philosophy, 107, 445-468.

Sedlár, I. (2019). Hyperintensional logics for everyone. Synthese. https://doi.org/10.1007/s11229-01802076-7.

Soames, S. (2019). Propositions as cognitive acts. Synthese, 196, 1453-1473.

Tichý, P. (1986). Constructions. Philosophy of Science, 53, 514-534.

Publisher's Note Springer Nature remains neutral with regard to jurisdictional claims in published maps and institutional affiliations. 